Revue internationale P.M.E.

Économie et gestion de la petite et moyenne entreprise

Revue

internationale

PME

\title{
Le management africain Mythe ou réalité
}

\section{Amadou Lamine Dia}

Volume 4, numéro 1, 1991

URI : https://id.erudit.org/iderudit/1008024ar

DOI : https://doi.org/10.7202/1008024ar

Aller au sommaire du numéro

Éditeur(s)

Presses de l’Université du Québec

ISSN

0776-5436 (imprimé)

1918-9699 (numérique)

Découvrir la revue

Citer cet article

Dia, A. L. (1991). Le management africain Mythe ou réalité. Revue internationale P.M.E., 4(1), 29-48. https://doi.org/10.7202/1008024ar

\section{Résumé de l'article}

Le management africain, tel qu'il existe aujourd'hui, est essentiellement caractérisé par un style de direction traditionnel (les dirigeants agissent au nom du groupe dont ils sont issus et pour lui) et personnel (autorité et pouvoir de décision se trouvant concentrés entre les mains d'un chef d'entreprise omniprésent). Il est la cause principale de l'échec des entreprises en Afrique, trop souvent peu rentables, non compétitives et éphémères. Il devrait donc ne devenir qu'un mythe et céder rapidement la place à un nouveau et véritable management africain qui, sans nier les spécificités culturelles de l'Afrique, les conjuguerait avec les principes de rigueur, de rationalité et de méthode, lesquels sont encore caractéristiques d'une gestion de type occidental, mais dont la valeur et l'efficacité doivent être considérées comme universelles.
Ce document est protégé par la loi sur le droit d'auteur. L'utilisation des services d'Érudit (y compris la reproduction) est assujettie à sa politique d'utilisation que vous pouvez consulter en ligne.

https://apropos.erudit.org/fr/usagers/politique-dutilisation/ 


\title{
Le management africain Mythe ou réalité
}

\author{
Amadou Lamine DIA* \\ Université Cheik H Anta Diop \\ Dakar
}

\begin{abstract}
RÉSUMÉ
Le management africain, tel qu'il existe aujourd'hui, est essentiellement caractérisé par un style de direction traditionnel (les dirigeants agissent au nom du groupe dont ils sont issus et pour lui) et personnel (autorité et pouvoir de décision se trouvant concentrés entre les mains d'un chef d'entreprise omniprésent). Il est la cause principale de l'échec des entreprises en Afrique, trop souvent peu rentables, non compétitives et éphémères. II devrait donc ne devenir qu'un mythe et céder rapidement la place à un nouveau et véritable management africain qui, sans nier les spécificités culturelles de l'Afrique, les conjuguerait avec les principes de rigueur, de rationalité et de méthode, lesquels sont encore caractéristiques d'une gestion de type occidental, mais dont la valeur et l'efficacité doivent être considérées comme universelles.
\end{abstract}

\begin{abstract}
African management today, essentially characterized by a traditional style of management (whereby managers act on behalf of and for the group from which they originated) as well as a personal style of management (whereby authority and decision-making are concentrated in the hands of an omnipresent company head) is the main reason for the failure in Africa of companies which too often are unviable, non-competitive and short-lived. This type of management should therefore disappear and be rapidly replaced by a new and real African management, which though not denying African cultural specificities, would associate them with principles of rigour, rationality and method. These principles characterize western management, but their value and efficiency must be regarded as universal.
\end{abstract}

* Monsieur Amadou Lamine Dia, maître de conférences en sciences de gestion à ENSUT, est membre du Comité technique spécialisé (Économie et gestion du CAMES) et directeur de l'École nationale supérieure universitaire de technologie (ENSUT) à Dakar. Adresse : ENSUT, B.P. 5085, Dakar-Fann. 


\section{RESUMEN}

La empresa africana tel como existe hoy, esencialmente carecterizada por un estilo de direccion traditional (los dirigentes obran en nombre del cual provienen y tambien para el) y personal (autoridad y poder de decision estan concentrados entre las manos de un jefe de empresa omnipresente) es la causa principal del fracaso de las empresas en Africa, muy a menudo poco productivas, no competitivas y efimeras. Debena dejar de ser un mito y ceder rapidamente la plaza a una nueva y verdadera empresa africana, que, sin negar las especifidades, y metodo aun y sobre todo caracteristicas de una gestion de tipo occidental, pero cuyo valor $y$ eficacidad han de ser considerados como universales.

\section{Introduction}

Le management, science de la gestion des entreprises, a vu le jour dans le contexte américain au lendemain de la Seconde Guerre mondiale. Les techniques expérimentées ont très rapidement fait l'objet d'une systématisation et d'une modélisation destinées à permettre leur application à des milieux socioculturels différents. Elles ont ainsi été diffusées vers le Japon et vers les pays européens, puis progressivement vers les pays en développement.

Le transfert des techniques de gestion s'est effectué à la fois par l'intermédiaire des consultants et des gestionnaires européens appelés dans le cadre de contrats techniques et grâce à des écoles de formation spécialisées. Cependant, bien que le problème de l'existence d'un management africain ait été à plusieurs reprises posé par les praticiens ${ }^{1}$, les travaux de recherche théorique sur la gestion de l'entreprise en Afrique demeurent encore trop peu nombreux.

C'est donc dans ce contexte que la présente étude définira les composantes essentielles d'un futur management spécifique à l'Afrique en les analysant dans une perspective diachronique.

1. On peut lire la synthèse des actes de colloques sur le management africain dans les revues suivantes : Le management a-t-il un avenir en Afrique ?, Marchés tropicaux et méditerranéens, $\mathrm{n}^{\circ} 2041,1984$, p. $3199-3200$; "La recherche des bons principes du management en Afrique », Marchés tropicaux et méditerranéens, $\mathrm{n}^{\circ} 2063,1985$, p. 1 273-1 274 ; « Marketing pour le développement », Revue française du marketing, $\mathrm{n}^{\circ} 112,1987 / 2$, p. 73. 


\section{De la culture africaine à un management spécifique}

L'étude d'un management particulier aux pays africains présuppose une définition de la culture africaine et une typologie des entreprises.

\subsection{Culture africaine et types d'entreprises}

\subsubsection{Le concept de culture}

Dans la langue française du Moyen Âge, le terme «culture " était synonyme de culte religieux. Le mot s'est ensuite appliqué au travail de la terre et, par métaphore, au domaine de l'esprit pour désigner à partir du XVIIr siècle le progrès intellectuel d'un individu.

L'acceptation moderne du mot a sans doute une origine allemande. JohanChristophe Adelung, par exemple, publie en 1782 un ouvrage sur l'histoire de la culture de l'espèce humaine dans lequel le terme "culture " prend le sens élargi de "progrès intellectuel et social de l'humanité ». Les phénomènes de groupes et de sociétés sont alors intégrés dans la création et l'évolution de la culture.

L'anthropologiste E.B. Tylor, dans un ouvrage publié en 1871 et intitulé Primitive Culture, donne une définition ethnographique du mot culture, encore d'actualité :

La Culture ou la Civilisation, entendue dans un sens ethnographique large, est cet ensemble complexe qui comprend les connaissances, les croyances, l'art, le droit, la morale, les coutumes et toutes les autres aptitudes et habitudes qu'acquiert l'homme en tant que membre d'une société.

C'est grâce aux sociologues américains Park, Burgess et Burn que le concept de culture, presque complètement ignoré par Marx, Weber et Durkheim, pénètre le domaine de la sociologie.

La transformation de la description antérieure de la culture en concept opératoire conduit à la définition suivante : la culture d'une nation est un ensemble cohérent de manières de penser, de sentir et d'agir, plus ou moins formalisé ayant pour but la survie et le développement de ses habitants.

Elle est en général forte, non seulement parce qu'elle se loge dans l'esprit des individus, mais aussi parce qu'elle est partagée par un grand nombre de personnes et qu'elle s'est cristallisée dans les institutions bâties par ces communautés : familles, système d'éducation, organisation des religions, formes de gouvernement, organisation du travail, lois, littérature, etc. 
Toutefois il faut noter que cette culture peut évoluer au contact des autres cultures et que la mondialisation des moyens de communication ainsi que le développement de la logistique affaiblissent les barrières culturelles.

\subsubsection{La culture africaine}

Compte tenu de la définition précédente, on devrait parler de cultures nationales, à l'instar de Henry Bourgoin (1984) et Desaunay (1979), dans leurs études sur la culture nationale de la Côte-d'Ivoire, car l'Afrique est composée de pays ayant chacun une culture propre.

L'existence d'une culture africaine définie comme un ensemble de croyances et de valeurs communes aux populations qui habitent l'Afrique, sans toutefois caractériser chaque individu, est cependant indéniable ${ }^{2}$. Les Africains ont le plus souvent un désir de " considération » et une volonté de s'extérioriser plus vifs que les Européens. Par exemple, c'est le désir de paraître et la volonté de se rehausser aux yeux de son groupe d'appartenance qui incitent bon nombre de dirigeants à fléchir devant les nombreuses sollicitations.

Les Africains sont également conditionnés par des influences culturelles de diverses natures imposées par la famille, le clan, l'ethnie et la caste.

\section{La famille ou le groupe}

Par «famille», il faut entendre la famille élargie regroupant les descendants d'un ancêtre commun et soudés entre eux par les liens du sang et du sol. Le groupe familial peut, du fait de la polygamie, devenir quantitativement très important. Il devient alors un clan à patronyme commun. Il s'organise autour d'un chef à la fois autoritaire et protecteur: l'homme le plus âgé de la famille, qui sert d'intermédiaire entre le monde des vivants et celui des morts, symbolise ainsi l'une des principales caractéristiques de la société africaine. Cette caractéristique confère au chef une autorité très grande, mais jamais despotique, sur les autres membres du groupe.

Cette forme d'organisation sociale n'admet pas d'individu isolé : ce dernier est avant tout le parent et le concitoyen d'un grand nombre de personnes. La conscience qu'il peut avoir de sa personne, de sa personnalité propre lui apparaît comme un phénomène tout à fait secondaire. La solidarité familiale est une contrainte imposée à tous les membres du groupe.

2. On peut citer, parmi les travaux les plus récents sur les caractéristiques de la culture qui déterminent un modèle d'organisation original propre au pays étudié, ceux de Geert Hofstede (1987). 


\section{口 Le système d'autorité}

Comme le souligne fort remarquablement Guy Desaunay (1979), l'autorité n'est, en Afrique, liée ni à la personne, ni à la compétence mais au statut social et au statut magique. En effet, est chef celui qui « sait », qui a « pouvoirs » et « bonne parole ». C'est dire que son autorité est sans appel.

Le champ d'application de la culture se prolonge jusqu'à l'entreprise industrielle, d'où la nécessité d'étudier le mode de fonctionnement des entreprises africaines.

\subsubsection{Adéquation entre culture et types d'entreprises}

Le tissu économique, composé d'un ensemble d'unités productives, est, en Afrique, d'une extrême diversité. Les entreprises africaines sont très différentes les unes des autres.

Il est cependant possible de les répartir en divers groupes définis par des caractéristiques homogènes. Plusieurs typologies satisfaisantes ont été proposées dans le cadre d'études sur les entreprises nationales. Dans le secteur primaire, le commerce, l'industrie ou les services, différentes catégories d'entreprises peuvent être distinguées selon un degré croissant de complexité : entreprise indépendante, artisanale, petite, moyenne, grande, société étrangère ou multinationale, société paraétatique ou société d'économie mixte.

En se fondant sur les caractéristiques majeures de leur organisation (statut, salariés permanents, secteurs d'activité), il est possible de construire les profils d'entreprises suivants : 


\section{TABLEAU 1}

Typologie des entreprises et caractéristiques majeures de leur organisation

\begin{tabular}{|c|c|c|c|}
\hline Caractéristiques & Statut & Secteurs d'activités & $\begin{array}{l}\text { Nombre de salariés } \\
\text { permanents }\end{array}$ \\
\hline INDÉPENDANT & $\begin{array}{l}\text { Société } \\
\text { familiale ou } \\
\text { indépendante }\end{array}$ & $\begin{array}{l}\text { Services, commerce de détail } \\
\text { parfois commerce de gros, } \\
\text { artisanat }\end{array}$ & Une dizaine au maximum \\
\hline $\begin{array}{l}\text { ENTREPRISE } \\
\text { ARTISANALE }\end{array}$ & $\begin{array}{l}\text { Société } \\
\text { familiale } \\
\text { ou associée }\end{array}$ & $\begin{array}{l}\text { Commerce de détail et de gros, } \\
\text { import-export, services, } \\
\text { petites unités industrielles }\end{array}$ & $\begin{array}{l}\text { Moins d'une vingtaine le } \\
\text { plus souvent }\end{array}$ \\
\hline $\begin{array}{l}\text { PETITE } \\
\text { ENTREPRISE }\end{array}$ & $\begin{array}{l}\text { Société de } \\
\text { personnes }\end{array}$ & $\begin{array}{l}\text { Principalement industrie, } \\
\text { travaux de construction, } \\
\text { travaux publics }\end{array}$ & Moins d'une trentaine \\
\hline $\begin{array}{l}\text { MOYENNE } \\
\text { ENTREPRISE }\end{array}$ & $\begin{array}{l}\text { Société de } \\
\text { personnes ou } \\
\text { société }\end{array}$ & $\begin{array}{l}\text { Commerce et surtout } \\
\text { industrie d'import-export }\end{array}$ & $\begin{array}{l}\text { Peut aller jusqu'à } \\
100 \text { salariés }\end{array}$ \\
\hline $\begin{array}{l}\text { GRANDE } \\
\text { ENTREPRISE }\end{array}$ & $\begin{array}{l}\text { Société } \\
\text { anonyme }\end{array}$ & $\begin{array}{l}\text { Grande distribution, } \\
\text { industries et services }\end{array}$ & $\begin{array}{l}\text { Nombre important } \\
\text { dépassant parfois } \\
\text { le millier }\end{array}$ \\
\hline $\begin{array}{l}\text { SOCIÉTÉ } \\
\text { ETRANGËRE } \\
\text { OU MULTI- } \\
\text { NATIONALE }\end{array}$ & $\begin{array}{l}\text { Société } \\
\text { anonyme }\end{array}$ & $\begin{array}{l}\text { Principalement commerce, } \\
\text { industrie de sous-traitance }\end{array}$ & $\begin{array}{l}\text { Nombre très variable } \\
\text { selon les activités }\end{array}$ \\
\hline $\begin{array}{l}\text { SOCIETÉ } \\
\text { PARAETATIQUE } \\
\text { OU SOCIETÉ } \\
\text { D'ECONOMIE } \\
\text { MIXTE }\end{array}$ & $\begin{array}{l}\text { Sociétés d'État } \\
\text { avec forte } \\
\text { participation } \\
\text { d'un ministère } \\
\text { de tutelle }\end{array}$ & $\begin{array}{l}\text { Secteurs considérés comme } \\
\text { stratégiques par l'État, aussi } \\
\text { bien du commerce, des } \\
\text { services, de l'industrie ou } \\
\text { du secteur rural }\end{array}$ & $\begin{array}{l}\text { En général en grand } \\
\text { nombre dépassant le } \\
\text { millier }\end{array}$ \\
\hline
\end{tabular}

Source : Tableau synthétique établi à partir des enquêtes sur les entreprises africaines : Senégal 1984 : Côte-d'Ivoire 1985, Cameroun 1985 ; Centre-Afrique 1984.

Les caractéristiques d'organisation se rapprochent donc dans l'ensemble de celles de l'entreprise européenne. La remarque s'applique surtout aux entreprises africaines affiliées à des structures multinationales.

En revanche, les petites entreprises dans lesquelles l'entrepreneur joue un rôle primordial sont nettement moins structurées. 


\subsection{Culture africaine et styles de direction}

Le style de direction est défini, en général, comme la manière dont les dirigeants d'une entreprise se conduisent pour atteindre leurs objectifs. Toutefois, le style de direction est ici conçu comme l'ensemble des méthodes et des processus de décision, des attitudes et des motivations de l'équipe dirigeante ou du dirigeant qui détermine le fonctionnement et les directions de l'entreprise.

Cette dernière définition permet de faire une synthèse du profil des entreprises africaines (tableau 2).

Les caractéristiques principales de styles de direction des entreprises africaines sont analogues à celles qui existent en Europe mais, plus la dimension de la firme est réduite, plus elles s'en éloignent.

La PME est le champ favorable d'expérimentation du management africain : les influences culturelles y sont plus nettes surtout sur le plan de la prise de décision et dans l'administration des hommes.

\subsubsection{L'administration}

\section{- Les hommes et leurs motivations}

Les anciens colonisateurs ayant surtout formé en pays anglophones des commerçants et en pays francophones des administrateurs, les dirigeants d'entreprises africaines ont été, et sont encore, trop peu nombreux. Et la situation est aggravée par l'impossibilité d'affecter les cadres originaires d'un pays d'Afrique dans un autre pays du continent. Les patrons africains aujourd'hui âgés de 50 ans et plus ont presque tous été des fonctionnaires et beaucoup, par prudence, le demeurent encore. Force est enfin de constater que les cadres africains ont dans leurs pays une disponibilité restreinte à cause des contraintes familiales, ethniques et politiques qui pèsent sur eux.

L'étude des motivations est un domaine en plein essor qui occupe une place importante dans les décisions des entreprises, des organismes publics, des sociétés à but non lucratif et des représentants élus des populations.

Les pays industrialisés ont manifesté plus d'intérêt et de détermination à étudier les motivations des hommes. En Afrique, le comportement et la motivation constituent un domaine de recherche assez nouveau (Desaunay, 1987; ENSUT, 1983). Le travail en Afrique est non seulement un moyen de gagner sa vie, mais aussi et surtout, une réponse au besoin de valorisation sociale de l'individu. Son salaire et son statut lui permettent de « rembourser » sa dette sociale et ainsi de conserver le soutien du groupe. 
TABLEAU 2

Typologie des entreprises et caractéristiques majeures de leurs styles de direction

\begin{tabular}{|c|c|c|c|c|}
\hline Caractéristiques & $\begin{array}{l}\text { Formation du directeur } \\
\text { Typologie }\end{array}$ & Mode de gestion & Cadre de gestion & Prise de décision \\
\hline Indépendant & $\begin{array}{l}\text { Directeur souvent illettré, } \\
\text { formation « sur le tas ». }\end{array}$ & $\begin{array}{l}\text { Autocratique et } \\
\text { « traditionnel ». }\end{array}$ & $\begin{array}{l}\text { Inexistant. Dans le système } \\
\text { traditionnel, un membre de la } \\
\text { famille conseille et influence } \\
\text { le directeur. }\end{array}$ & $\begin{array}{l}\text { Prise sur la base de la caisse, } \\
\text { des perspectives de récupérer } \\
\text { son argent ou de gagner } \\
\text { " gros » rapidement. }\end{array}$ \\
\hline $\begin{array}{l}\text { Entreprise } \\
\text { artisanale }\end{array}$ & $\begin{array}{l}\text { Technique professionnelle } \\
\text { ou apprentissage du métier } \\
\text { « sur le tas ». }\end{array}$ & $\begin{array}{l}\text { Autocratique et } \\
\text { « traditionnel ». }\end{array}$ & $\begin{array}{l}\text { Techniciens diplômés s'occu- } \\
\text { pant essentiellement de la } \\
\text { production. }\end{array}$ & $\begin{array}{l}\text { Prise par le dirigeant sur la } \\
\text { base des besoins de sa produc- } \\
\text { tion et de son « flair » du } \\
\text { marché. }\end{array}$ \\
\hline $\begin{array}{l}\text { Petite entre- } \\
\text { prise }\end{array}$ & $\begin{array}{l}\text { Formation professionnelle } \\
\text { et/ou « sur le tas ». }\end{array}$ & « Traditionnel ». & $\begin{array}{l}\text { Surtout des techniciens. L'un } \\
\text { d'eux, par exemple le compta- } \\
\text { ble, peut jouer un rôle } \\
\text { privilégié pour conseiller le } \\
\text { directeur. }\end{array}$ & $\begin{array}{l}\text { Les principales sont prises par } \\
\text { l'entrepreneur, les décisions } \\
\text { techniques courantes, par le } \\
\text { chef d'atelier, mais sanction- } \\
\text { nées par le directeur. }\end{array}$ \\
\hline $\begin{array}{l}\text { Moyenne } \\
\text { entreprise }\end{array}$ & $\begin{array}{l}\text { En général de formation } \\
\text { supérieure. } \\
\text { DUT, ingénieur. }\end{array}$ & $\begin{array}{l}\text { De moins en moins } \\
\text { traditionnel. La structure et } \\
\text { les problèmes plus } \\
\text { complexes imposent un } \\
\text { degré de participation plus } \\
\text { ou moins important. }\end{array}$ & $\begin{array}{l}\text { De plus en plus nombreux et } \\
\text { indispensable, complexité en } \\
\text { raison d'une croissance : } \\
\text { comptables, agents commer- } \\
\text { ciaux. En général un début } \\
\text { d'organisation clarifie les } \\
\text { rôles, les responsabilités et les } \\
\text { pouvoirs. }\end{array}$ & $\begin{array}{l}\text { La décision finale est prise par } \\
\text { l'entrepreneur, mais il demande } \\
\text { souvent l'avis de ses cadres ou } \\
\text { des techniciens. }\end{array}$ \\
\hline
\end{tabular}


Tableau 2 (suite)

Typologie des entreprises et caractéristiques majeures de leurs styles de direction

\begin{tabular}{|c|c|c|c|c|}
\hline Caractéristiques & $\begin{array}{c}\text { Formation du directeur } \\
\text { Typologie }\end{array}$ & Mode de gestion & Cadre de gestion & Prise de décision \\
\hline $\begin{array}{l}\text { Grande } \\
\text { entreprise }\end{array}$ & $\begin{array}{l}\text { De plus en plus un } \\
\text { gestionnaion supérieure ; } \\
\text { au minimum un technicien } \\
\text { de haut niveau avec une } \\
\text { expérience professionnelle } \\
\text { importante. }\end{array}$ & $\begin{array}{l}\text { Exploitation des méthodes } \\
\text { modernes avec parfois un } \\
\text { système de concertation ou } \\
\text { de participation formel ou } \\
\text { informel. }\end{array}$ & $\begin{array}{l}\text { En général un personnel de } \\
\text { cadres assez important. } \\
\text { Existence d'un organigramme } \\
\text { et d'une structure hiérarchique. }\end{array}$ & $\begin{array}{l}\text { Souvent prise au sein d'organe } \\
\text { de gestion de type comité de } \\
\text { direction. }\end{array}$ \\
\hline $\begin{array}{l}\text { Société } \\
\text { étrangère ou } \\
\text { multinationale }\end{array}$ & $\begin{array}{l}\text { Expatrié : gestionnaire ou } \\
\text { technicien avec expérience } \\
\text { en gestion. }\end{array}$ & Moderne. & Pour l'essentiel des activités. & $\begin{array}{l}\text { Se prend souvent à l'étranger } \\
\text { en concertation avec le délégué } \\
\text { sur place. }\end{array}$ \\
\hline $\begin{array}{l}\text { Société } \\
\text { paraétatique } \\
\text { ou société } \\
\text { d'économie } \\
\text { mixte }\end{array}$ & $\begin{array}{l}\text { Issue de la fonction } \\
\text { publique ; plutôt adminis- } \\
\text { trateur public ; parfois } \\
\text { technicien. }\end{array}$ & Moderne mais bureaucrate. & $\begin{array}{l}\text { Souvent en grand nombre en } \\
\text { raison des exigences d'un } \\
\text { certain contrôle. }\end{array}$ & $\begin{array}{l}\text { Processus souvent long et } \\
\text { hiérarchisé. Au niveau courant, } \\
\text { système de concertation. Selon } \\
\text { les pays, plus ou moins large } \\
\text { délégation au niveau des } \\
\text { directions, mais un contrôle } \\
\text { complexe par l'autorité de } \\
\text { tutelle. }\end{array}$ \\
\hline
\end{tabular}


Le personnel, dans la plupart des cas, n'a véritablement connaissance ni du fonctionnement de l'entreprise ni des contraintes qu'elle doit subir. Celles-ci demeurent donc extérieures et mal perçues. L'individu n'a aucun sentiment de culpabilité face à un travail mal fait ou même face à un échec. La contrainte que l'individu s'impose à lui-même et qui l'oblige, pour des raisons morales, à déployer tous ses efforts pour atteindre un résultat, n'existe pas et ne peut donc jouer le rôle moteur qu'elle joue dans les entreprises occidentales.

Dans les pays occidentaux, le système de compétition incite les cadres et les dirigeants à prendre des risques, à améliorer leur carrière, à rechercher les responsabilités. En Afrique, le besoin d'appartenance et de pouvoir prime sur celui de réalisation. Le cadre accède par sa fonction à un certain rang social et il cherche avant tout à conserver cette fonction en nouant de bonnes relations avec son patron et avec les autres agents. Sa contribution à la réussite de l'entreprise et son autoréalisation n'ont qu'une faible importance au regard de ce souci constant.

Esprit d'entreprise et goût d'entreprendre ne sont certes pas inconnus en Afrique, mais ils semblent orientés vers la recherche d'un statut social ou vers la spéculation.

Le patron exerce une attraction réelle sur l'ensemble du corps social de son entreprise mais le potentiel de loyauté et de motivation ainsi disponible est lié à l'homme. Certains dirigeants le cultivent en amplifiant le côté relationnel, devenant ainsi de véritables chefs de quartiers ou de village, rendant la justice, dispensant des conseils matrimoniaux. D'autres tentent d'obtenir un transfert de ce potentiel vers l'entreprise. Mais, faute d'identification, de symboles et de valeurs collectifs, ce transfert se fait en pure perte, entrainant frustration et démotivation.

Le patron possède, surtout dans les petites et moyennes entreprises, un pouvoir sans limite. Étant au cœur de l'entreprise, il coordonne toutes les actions et dirige selon sa vision et son flair.

\section{口 L'organisation}

À l'exception des grandes firmes, où les relations sont souvent formelles, la plupart des entreprises n'ont pas d'organigramme, et le rôle fonctionnel de chacun des agents n'est pas précisément défini. Ce sont généralement les circonstances qui dictent à l'individu sa conduite. Cette situation est du reste souvent entretenue, afin que toute tâche puisse, quelle qu'elle soit, être imposée à tous.

De plus, le recrutement du personnel est l'affaire quasi privée du chef d'entreprise, qui a, par là même, tendance à recruter les membres de sa famille, de son ethnie, de son village. Recruter un membre du groupe est une obligation 
stricte à laquelle on ne peut échapper sans courir le risque de sanctions sociales graves (passage devant le tribunal de famille...), et de perdre ainsi un prestige considérable. Le système de commandement se trouve ainsi faussé. Et c'est ainsi que les dirigeants africains paraissent, aux yeux de leurs homologues étrangers, à la fois cassants vis-à-vis de leurs subordonnés et trop proches d'eux (Olomo, 1987; Desaunay, 1987). Il est effectivement difficile d'imposer le respect à des individus dont le choix est dicté par le groupe d'appartenance.

\subsubsection{La prise de décision}

Dans le contexte africain, le décideur essaie rarement de maîtriser les éléments extérieurs, qu'il considère comme supérieurs et échappant à son emprise. La planification des activités est souvent inconnue et les grandes décisions sont prises rapidement, sous la pression des circonstances. Un importateur, par exemple, ne mesure pas les évolutions possibles de la consommation, mais achète généralement dès que la rupture des stocks est proche ou s'il a des informations sur une probable pénurie à venir ${ }^{3}$.

Ainsi, la gestion par objectifs devient impossible, en raison d'une certaine réticence à l'égard de l'idée même de planification et d'un fatalisme évident particulièrement chez les musulmans, pour peu que la réalisation des objectifs soit entravée par des éléments extérieurs.

Le pouvoir se trouve concentré entre les mains de dirigeants dont l'omniprésence influence toutes les décisions. Il s'acquiert d'ailleurs par la force, ne se négocie ni ne se partage. Pour le conserver, les responsables établissent généralement une distance hiérarchique entre eux-mêmes et leurs collaborateurs immédiats et filtrent systématiquement la diffusion des informations. Ce n'est que par ce mécanisme de concentration des informations au sommet de la hiérarchie que la direction parvient à imposer son autorité.

Les relations entre la direction générale et les cadres supérieurs s'exercent exclusivement par l'intermédiaire des ordres d'exécution. Des flux vers le sommet sont possibles, mais le système ne les encourage pas et a tôt fait de démontrer qu'ils nuisent au bon fonctionnement des rouages de l'entreprise. La décision finale émane en définitive du groupe ou des leaders reconnus dans le groupe dont sont issus les dirigeants.

Tels semblent être le style de direction africain et la caractéristique d'un management propre à l'Afrique. Le transfert de technologie a seulement favorisé l'apparition, à côté de ce management traditionnel, personnel, relationnel et

3. On peut consulter la synthèse des Journées de réflexion sur le thème : Le marketing sénégalais, tenue à l'ENSUT, Université. Cheikh Anta Diop Dakar, février 1984. 
social, d'un management de type occidental, moderne, rigoureux, rationnel et méthodique. D'où la situation actuelle, caractérisée par la juxtaposition de deux modes opposés. Est-il possible de dépasser cette antinomie?

\section{Vers un nouveau management africain}

\subsection{Résultats pratiques du système actuel de gestion}

\subsubsection{La création d'entreprises et la compétitivité}

La création d'entreprises, particulièrement d'unités industrielles, peut avoir un effet bénéfique, tant sur la structure de la production intérieure brute que sur la croissance et le développement économique et social. Les pays d'Afrique ont donc fait le choix de politiques industrielles privilégiant les petites et moyennes entreprises. Il faudrait cependant, pour lutter avec efficacité contre le sousdéveloppement, que ces entreprises soient bien gérées et compétitives.

Or, l'expérience des 25 dernières années montre qu'en dépit de quelques véritables succès, la situation générale des entreprises est loin d'être brillante : taux de mortalité important (6 entreprises sur 10), croissance quasi insignifiante (ENSUT, 1987). Beaucoup, particulièrement les entreprises de petites et moyennes dimensions ainsi que les entreprises publiques sont dépourvues de toute efficacité sur le plan de leur fonctionnement et de leur performance. Pour certaines, la rentabilité est négative, pour d'autres, très médiocre (Causse, 1986).

\subsubsection{Les causes de l'insuccès}

Plusieurs explications peuvent être avancées. Les unes sont liées à l'histoire, d'autres à la conjoncture actuelle (chute de l'activité économique en raison de la sécheresse, des mauvaises récoltes, baisse des cours des principaux produits d'exportation, blocage des prix et des tarifs, endettement, concurrence d'autres pays, rareté des aides, répercussion de la crise internationale). D'autres, enfin, sont consécutives aux déficiences du management (les dirigeants d'entreprises ont pris en considération, dans la conduite des affaires, les seules techniques de gestion, au mépris du facteur culturel, ou ont, au contraire, accordé à ce dernier une attention exclusive).

Quelques exemples sont particulièrement significatifs (Gouadain, 1985 ; Causse, 1986 ; Delalande, 1987). 


\section{Dans le domaine de la gestion commerciale}

- De nombreux chefs d'entreprises estiment que la société africaine est encore une société de pénurie, dans laquelle la gestion commerciale ou le marketing sont des raffinements superflus. Ils subissent de lourdes pertes en vendant indistinctement aux Africains sans tenir compte de leurs différenciations sociologiques. Pourtant, la population africaine se répartit en cinq catégories principales : le secteur rural traditionnel, à économie de subsistance ou à forte autoconsommation; le secteur rural diversifié, fruit de la croissance de l'économie monétaire et du développement agricole et commercial ; le secteur urbain marginal ; le secteur urbain à revenu moyen ; celui des classes privilégiées, cancérisé par une consommation de masse de type occidental.

- En ce qui concerne la transposition sans discernement des méthodes d'études de marché pour la commercialisation de nouveaux produits, le résultat a été négatif ; en tout cas, il n'a pas répondu aux prévisions. D'une part, les statistiques sociodémographiques, de production, de consommation, d'importation... manquent, le plus souvent, de fiabilité. D'autre part, l'échantillonnage probabiliste, fondement des études qualitatives, est rendu hasardeux ou trop coûteux par l'inexactitude, où même l'inexistence des cartes et des plans de villes. Enfin, la collecte des données par téléphone ou par correspondance est quasi impossible et celle faite par des enquêteurs est entravée par leur petit nombre, la multiplicité des langues et les fréquents refus de réponse.

- Dans la conception des produits, les mobiles techniques sont privilegiés par rapport aux mobiles commerciaux, alors qu'une démarche de marketing logique devrait conduire l'entreprise à se définir constamment par rapport à son environnement et au marché des besoins individuels qu'elle a décidé de satisfaire. Des produits conçus sans tenir compte du goût des consommateurs africains ont en général une durée de vie courte (Boyer, 1983 ; Bollinger, 1977 ; Maricourt, 1987). Tel fut, par exemple, le cas du pamiblé (pain composé de $15 \%$ de mil et de $85 \%$ de blé) mis sur le marché au Sénégal et dont la fabrication a dû cesser au bout de trois mois parce que son goût ne plaisait pas aux consommateurs urbains.

- La politique de communication des entreprises africaines a souvent conduit à l'échec en raison soit de l'absence de distinction entre le segment riche de la population et les masses, soit de l'opinion de certains dirigeants accordant une efficacité absolue aux seules palabres, aux discours comiques ou au « matraquage » publicitaire. 
- La distribution des produits pose des problèmes extrêmement complexes aux entreprises, parce que les spécificités africaines ne sont pas cernées et prises en compte. Par exemple, la fonction de demi-grossiste a disparu dans les pays industrialisés, alors qu'en Afrique on trouve non seulement l'importateur et le grossiste, mais ensuite quatre ou cinq types de demi-grossistes, suivis d'une série de détaillants, de sousdétaillants, etc.; ce qui constitue un handicap sérieux pour la compétitivité des petites et moyennes entreprises commerciales.

- L'application d'une stratégie de prix en fonction de la position de la firme sur le marché s'avère difficile dans la mesure où la plupart des États imposent des prix pour la commercialisation des biens de consommation de première nécessité.

- Dans le domaine de la gestion du personnel et des œuvres sociales

- La formation insuffisante du personnel, ses motivations et ses comportements constituent autant d'obstacles au développement, et même à la simple survie de l'entreprise. La vue extrêmement parcellaire qu'ont les ouvriers, la plupart des employés et une partie des contremaîtres, de l'entreprise et des processus qui s'y déroulent ne permet pas d'appréhender l'influence du travail personnel sur les résultats d'ensemble (Desaunay, 1987 ; Bourgoin, 1984).

- Le recrutement du personnel de l'entreprise essentiellement fondé sur le critère de l'appartenance à une famille, une ethnie ou un clan ne favorise ni la motivation, ni l'émulation, ni la responsabilité. Et la productivité s'en trouve réduite.

- L'entreprise africaine ne peut être compétitive, car on attend seulement d'elle qu'elle soit au service de la solidarité des groupes représentés en son sein et de leurs clients.

- Une autorité reposant exclusivement sur la compétence ne pourra suffire à la bonne marche de l'entreprise si elle n'intègre pas les références culturelles de ceux sur qui elle s'exerce.

口 Dans le domaine de la gestion comptable et financière

- Dans ce domaine, il s'agit moins d'une inadéquation de l'outil de gestion comptable et financière que de sa mauvaise appreciation par les entreprises. En effet, les nombreux insuccès proviennent de la 
confusion entre le patrimoine de l'entreprise et celui de l'entrepreneur : dans les PME, le patron et sa famille font souvent des prélèvements en cas de besoin, dilapidant ainsi les ressources de l'entreprise.

En introduisant sans discernement les principes de rigueur et d'efficacité, on a ainsi défait la trame sociale et culturelle préexistante dans les pays africains. L'intrusion des méthodes occidentales de gestion a été la cause, dans les entreprises africaines, des " acculturations et des déséquilibres » (Kizerbo, 1976). Il apparaît, en définitive, que le management occidental n'a pas donné les résultats escomptés, c'est-àdire sortir les entreprises et les pays africains d'un sous-développement chronique.

\section{Réconciliation de la tradition et de la modernité}

\section{1. Échec du modèle occidental}

La transposition pure et simple du modèle occidental en Afrique ne peut qu'échouer : les valeurs, les cultures, l'environnement, tout est trop différent.

Les travaux de Peters et Waterman (1983), Mintzberg (1976), Koestler (1968), Tversky et Kahneman (1974) ont clairement montré que l'esprit et la mentalité sont conditionnés par le milieu social et culturel, que l'homme est très souvent guidé par son intuition ou son subconscient dans ses décisions et ses activités journalières. Le milieu intime a une incidence sur la vie professionnelle.

Lawrence et Lorsch (1973), dans leur théorie relativiste dite «théorie de la contingence » (la plus récente des théories de l'organisation) ont souligné qu'il n'existe pas de théorie unique et universellement valable, dans le temps comme dans l'espace, pour résoudre les problèmes des organisations. Les structures de l'entreprise doivent donc s'adapter à l'environnement ou à son évolution. C'est dans le cadre de cette théorie que nous allons tenter de définir le management africain de demain (D’Iribarne, 1985 ; Gupta, 1984 ; Cyert, 1970 ; Drucker, 1961 ; Ouchi, 1982).

Le nouveau management devra réconcilier tradition et modernité et tirer parti de l'une et l'autre pour faire de l'entreprise africaine une unité de production rentable et compétitive. La gestion en tant qu'outil de rentabilité dans les pays en développement est au stade de l'adolescence. Les hommes apprennent progressivement, au fil des expériences, à organiser, administrer, décider, c'està-dire gérer. L'Afrique, par là même, constitue une école de gestion maîtrisant les réalités de l'environnement et favorisant l'apprentissage des techniques ainsi que l'éclosion des talents. 
Le mode de gestion des entreprises africaines, tel qu'il existe maintenant, ne doit être qu'une étape vers l'utilisation d'un nouveau management africain, lequel comprendra les principes particuliers sur lesquels les chefs d'entreprises pourront agir pour rendre leurs structures performantes.

\subsection{Principes immuables du management}

Ces principes sont la rigueur, l'efficacité et la rentabilité. Il existe, en effet, un minimum de structures de comportements fondamentaux et universels que la culture ne saurait remettre en cause.

Que l'entreprise soit nord-américaine, asiatique, européenne ou africaine, qu'elle soit protégée ou exposée à la concurrence, elle doit tenir compte pour sa performance de trois données fondamentales :

- un marché, une clientèle et un produit ou un service qui présentent assez d'avantages pour permettre la satisfaction d'un besoin ;

- un personnel remplissant les diverses fonctions d'encadrement et d'exécution ;

- un capital destiné à l'acquisition des outils nécessaires à l'exploitation et au développement, et dont les possesseurs attendent en contrepartie, la rémunération et la valorisation.

Les entreprises africaines, au même titre que celles situées dans d'autres continents, doivent être rentables et solvables afin de survivre et de se développer à long terme.

Pour l'entreprise, la solvabilité consiste à assurer à tout instant le paiement de ses dettes exigibles, tandis que la rentabilité se définit comme l'aptitude à créer un bénéfice.

Le bénéfice est fondamental : il permet l'accumulation de liquidités suffisantes pour assurer le maintien du capital de production de la firme, assurer le remboursement des emprunts, contribuer à la naissance du capital nouveau et dégager une rémunération du capital investi par les associés. Cependant, l'objectif de rentabilité ne représente pas la même chose pour toute entreprise. D'une part, parce que les rémunérations personnelles et familiales jouent un rôle plus important dans les petites et moyennes entreprises que dans les grandes entreprises. D'autre part, parce que si l'on raisonne dans la grande entreprise en terme de rentabilité à moyen terme, c'est la recherche de la rentabilité à court terme qui prévaut pour les PME africaines.

Ainsi, les chefs d'entreprises africains, comme leurs homologues d'autres secteurs géographiques, ont pour tâche de satisfaire les aspirations de tous leurs partenaires, d'être en permanence responsables de cet équilibre et de veiller à le 
rétablir toutes les fois qu'il se trouve rompu, au risque de disparaitre tout en ruinant la collectivité. Ils ont le devoir de compter et le développement des pays africains suppose une gestion rationnelle et efficace des entreprises.

\subsection{Variables culturelles du management africain}

Tout en constituant des obstacles au développement des entreprises africaines, les variables culturelles peuvent, quand elles sont examinées avec discernement, offrir nombre d'occasions, comme le montrent les exemples suivants.

\section{口 Dans le domaine de la gestion commerciale}

- Les études de marché sont possibles en Afrique. Elles peuvent éviter bien des erreurs à condition que leurs auteurs se contentent de méthodes assez rudimentaires, appropriées et faisant largement appel au jugement et au bon sens. Il faut souvent utiliser des échantillonnages empiriques, recruter des enquêteurs en fonction des populations étudiées et les entraîner soigneusement, s'appuyer sur les notables et les chefs de famille et préparer les interviews par des explications simples et convaincantes.

- La politique de produit suppose, dans le contexte africain, une schématisation. Il faut proposer des produits simples et robustes, à longue duree de vie, pour tenir compte d'une part, des faibles possibilités de maintenance, d'autre part, du bas niveau des revenus. Les emballages doivent être renforcés en raison des difficultés de stockage et de transport et facilement réutilisables, éventuellement pour d'autres usages.

- La segmentation de la population en plusieurs catégories de consommateurs nécessite une diversité de conditionnement destinée à faciliter les achats en très petites quantités par les populations à faible pouvoir d'achat.

- En matière de prix, il convient de rechercher le prix le plus logique, le plus bas possible à la lumière du pouvoir d'achat des consommateurs. Les prix fixés par l'État s'imposent à la firme. Celle-ci doit donc accorder une attention particulière à la procédure publique de détermination des prix. Par ailleurs, la firme doit disposer d'une comptabilité analytique permanente et rigoureuse pour être en mesure de présenter un dossier de structure de coûts et de prix de revient corrects. Cette précaution permet d'éviter que le prix de vente fixé par les pouvoirs publics sur la base de ce dossier ne couvre pas les coûts de l'entreprise. 
- Dans le domaine de la gestion du personnel et des affaires sociales

- Les conflits entre l'impératif industriel et l'impératif traditionnel peuvent être écartés par une bonne gestion des affaires sociales. Il en est ainsi des relations financières entre le dirigeant d'entreprise et son personnel. Au nom de la solidarité, le patron offre souvent à ses employés des sommes allant de 1000 à 25000 Frs CFA, puisées dans la caisse de l'entreprise et sans remboursement.

- Une solution peut être trouvée sur le modèle d'une pratique déjà courante dans de nombreuses entreprises, où les salariés se sont organisés eux-mêmes spontanément en une sorte de « tontine »: chacun des membres adhérents verse chaque mois la même somme d'argent et l'un d'entre eux bénéficie de la totalité de la somme collectée, ce qui lui permet d'utiliser une somme importante pour faire face à une dépense imprévue qu'il n'aurait pu assumer seul.

- De la même manière, un fonds de solidarité communautaire peut être constitué officiellement dans le but de couvrir des dépenses familiales exceptionnelles des membres du personnel.

\section{$\square$ Dans le domaine de la gestion comptable et financière}

- Le redressement de l'entreprise africaine passe par l'amélioration de son fonds de roulement et, par voie de conséquence, du renforcement des fonds propres. C'est au démarrage même de l'entreprise qu'il faut réunir des fonds propres suffisants. La constitution d'une entreprise sociétaire ou collective s'avère donc nécessaire, car le promoteur africain est généralement peu fortuné. Toutefois, il est souvent difficile de convaincre un créateur d'entreprise africain qu'il doit accepter d'être minoritaire s'il veut réussir.

- L'utilisation d'instruments fiables, légers et adaptés constitue l'autre élément d'une bonne gestion financière. On lit parfois que la comptabilité dans les entreprises africaines est approximative ou inexistante. C'est souvent vrai dans le petit commerce ou l'artisanat, mais inexact dans l'entreprise industrielle. Par contre, ce qui est fréquent, c'est le délai excessif de traitement des informations comptables. Les états financiers sont produits trop peu fréquemment et trop tardivement pour servir d'instruments efficaces de gestion. Un peu de rigueur dans le service comptable pourrait souvent remédier à ce défaut plus sûrement que l'introduction de l'informatique. 


\section{Conclusion}

L'Afrique a été pendant longtemps connue à travers ses militaires, ses hommes politiques et quelques hauts fonctionnaires. Les gestionnaires africains commencent à entrer en scène et il est à souhaiter qu'ils puissent prendre, dans les années à venir, la place qui leur revient dans le développement économique et social de leur continent. L'Afrique peut se développer sans perdre son âme, à condition que ses dirigeants $y$ introduisent une forme particulière de management jusqu'à présent trop souvent ignorée. Tourné vers l'extérieur, ce management devrait, pour réussir, avoir une certaine spécificité culturelle, sans toutefois conférer aux particularités locales africaines une valeur intangible et sacree ni remettre en question les principes de base universels.

Avec ses imperfections et ses tares, l'actuel mode de gestion des entreprises devrait disparaître. Le nouveau management africain tel que nous l'avons défini n'est encore qu'un mythe, mais il serait souhaitable qu'il puisse, dans les plus brefs délais, devenir une réalité.

\section{Bibliographie}

Bamderanbako, D. (1985), Mode de gestion des P.M.E. au Cameroun, Bujumbura (Centre universitaire de recherche pour le développement économique et social, CURDES).

Bollinger, D. (1977), Le marketing en Afrique, tome 1 : La Côte d'Ivoire, Abidjan, Éd. CEDA.

Bourgorn, H. (1984), L'Afrique malade du management, Paris, Éd. Jean Picollec (Perspective 2001).

BOYER, A. (1983), "African premarketing ", XII ${ }^{\text {th }}$ Annual conference of the European marketing Academy, Grenoble, avril.

CAUSSE, G. (1986), Les obstacles à la gestion de type privé dans les entreprises publiques africaines, colloque international et interdisciplinaire sur le thème «Entreprise publique et développement » (Cotonou, 24-26 février 1986), publication du Laboratoire de gestion et de culture africaines, Nice, 1986.

Cyert, R. et J.G. March (1970), Processus de décision dans l'entreprise, Paris, Dunod. Delalande, P. (1987), Gestion de l'entreprise industrielle en Afrique, Paris, Economica.

Desaunay, G. (1979), «Les sciences humaines, c'est quoi ? Réflexion sur une expérience d'enseignement des sciences humaines dans les écoles de gestion d'Abidjan ", les Cahiers de recherches du CESA (HEC), $n^{\circ} 124$.

Desaunay, G. (1985), «Les relations humaines dans les entreprises ivoiriennes », Revue française de gestion, $n^{\circ} 64$, p. 95-101.

Desaunay, G. (1987), Gestion du personnel : outil ou clef de la réussite, thème des journées de réflexion de l'ENSUT, Université Cheikh Anta Diop (Dakar), mars. 
DiA, A.L. (1984), «Choix stratégique de l'entreprise privée sénégalaise », Afrique et Développement, octobre-décembre , p. 33-48.

D'IRIBARNE, P. (1985), « La gestion à la française », Revue française de gestion, janvierfévrier, p. 5-13.

DRUCKER, P.F. (1961), The Patrice Management, London, Mercury Books.

Gouadain, D. et G. Le Contre (1985), Difficultés de gestion des entreprises des pays en développement, Laboratoire de gestion et de culture, Nice, France, p. 7.

Gupta, J. (1984), «Le style de management indien», Revue française de gestion, janvier-février, p. 80-85.

Hofstede, G. (1987), « Relativité culturelle des pratiques et théories de l'organisation », Revue française de gestion, $\mathrm{n}^{\circ} 64$, septembre-octobre, p. 10-21.

KIZERBo, J. (1976), « Culture et Développement », Institut international d'études sociales, $n^{\circ} 8$, Genève.

KoEstLer, A. (1968), Le cheval dans la locomotive; le paradoxe humain, Paris, Éd. Calman-Lévy.

Kofri Kouadio, D. (1983), La création d'entreprises privées par les nationaux en Côte d'Ivoire depuis 1960, Abidjan, Éd. CEDA.

LAWRENCE, P.R. et J. W. LORSCH (1973), Adapter les structures de l' entreprise. Intégration ou différenciation, Paris, Éd. d'organisation.

MARICOURT, R. de (1987), « Les principes et techniques de marketing sont-ils applicables aux pays en développement ? ", Revue française de marketing, $\mathrm{n}^{\circ} 112$.

MintzBeRg, G. (1976), «Planning on the left side and making on the right », Harvard Business Review, juillet- août, p. 53.

Olomo, P.R. (1987), «Comment concilier tradition et modernité dans l'entreprise africaine », Revue française de gestion, $\mathrm{n}^{\circ} 64, \mathrm{p} .91-94$.

Ouch, W. (1982), Théorie Z : faire face au défi japonais, trad. J. Guiod, Paris, Interéditions.

Peters, T. et R. Waterman (1983), Le prix d'excellence, Paris, Ed du Club France Loisirs.

TVERSKy A. et D. KAHNEMAN (1974), «Judgment under uncertainty: heuristics and biases », Sciences, 27 septembre, p. 1124. 